\title{
Clinicopathology Figures and Long-term Effects of Tamoxifen Plus Radiation on Survival of Women with Invasive Ductal Carcinoma and Triple Negative Breast Cancer
}

\author{
Mehrdad Payandeh ${ }^{1}$, Masoud Sadeghi $^{2 *}$, Edris Sadeghi $^{2}$, Mehrnoush Aeinfar $^{2}$
}

\begin{abstract}
Background: Triple negative breast cancer (TNBC), characterized as estrogen receptor (ER), progesterone receptor (PR) and human epidermal growth factor receptor 2 Her2 negative and accounting for 10-17\% of all breast carcinomas, is only partially responsive to chemotherapy and suffers from a lack of clinically established targeted therapies. The aim of the current study was to evaluate the patterns of treatment and clinicopathology figures in Kurdish patients with triple-negative breast cancer, and to compare these to other reports. Materials and Methods: Between 2001 and 2014, 950 breast cancer patients were referred to our clinic. There were 74 female patients with TNBC, including 70 patients was invasive ductal carcinoma entered into our study. ER and PR positivity was defined as positive immunohistochemical staining in more than $10 \%$ of tumor cells. Immunohistochemistry assay with anti-HER2 antibodies was used to identify HER negative (0 and 1+) or positive (2+ and 3+). HER2 gene amplification was determined by fluorescent in situ hybridization (FISH). Overall survival (OS) was plotted with GraphPad Prism 5 Software using Kaplan-Meier and log-rank tests for comparison of results. Results: The mean age in the first diagnosis for 70 patients with triple TNBC and invasive ductal carcinoma was 49.6 years that range of age was 27-82 years. All of the patients were female. Of 70 patients, 23 patients had metastasis. Thirty-two patients $(45.7 \%)$ were treated with tamoxifen and 39 $(55.7 \%)$ with radiotherapy. Three-year, 5-year and 10-year OS rates for all patients were $82 \%, 72 \%$ and $64 \%$, respectively. Conclusions: The OS in our West Iran TNBC patients is less than reported elsewhere. However, treatment with combination of tamoxifen plus radiation increases the OS and reduces the mortality rate.
\end{abstract}

Keywords: Overall survival - radiation - tamoxifen - TNBC - Kurdish Iran

Asian Pac J Cancer Prev, 16 (12), 4863-4867

\section{Introduction}

Invasive breast carcinoma is the most common malignant tumor in women worldwide (Dogra et al., 2014) and is a heterogeneous disease with distinct pathological and histological features, and can be classified into several subtypes based on the expression of 3 receptors: estrogen receptor (ER), progesterone receptor (PR), and human epidermal growth factor receptor 2 (HER2/ neu). The breast tumors that lack the expression of all these receptors are termed triple-negative breast cancers (TNBC) (Li et al., 2013). ER positivity predicts response to endocrine therapy such as antiestrogen (tamoxifen) and trastuzumab therapy (Herceptin) for tumor with HER2/ neu overexpression (Sharma et al., 2014). Trastuzumab therapy is effective and improves the survival of HER2positive breast cancer patients (Payandeh et al., 2014). The triple negative subgroup of breast cancer has been reported to have different incidence among different ethnic groups and have been associated with lower disease-free survival, a higher predisposition to visceral metastases and poorer outcome when compared to other subtypes of breast cancer (Tan et al., 2009). Higher rates of TNBC have been observed in women who are younger, and also current treatment strategies include many chemotherapy agents, such as the anthracyclines, taxanes, ixabepilone, and platinum agents, as well as selected biologic agents and possibly anti-EGFR drugs (Hudis et al.,2011) but with chemotherapy alone the residual risk remains substantially higher, between $30 \%$ and $40 \%$. Improving outcomes in triple-negative disease will require better understanding of the biology and drug targets in this subtype (Carey, 2011). The shape of the survival curve for patients with triple-negative or basal-like breast cancer differs from that for patients with other types of breast cancer: there is a sharp decrease in survival during the first 3 to 5 years after diagnosis, but distant relapse after this time is much less common and After 10 years, relapse is more likely among patients with ER-positive cancers than among patients with ER-negative cancers (Foulkes et al., 2010). An alternative hypothesis is that variants or even combinations of variants in the TNBC associated risk loci 
may act to change existing malignant breast lesions to a TN phenotype during the formation of the tumor (Stevens et al., 2013).

The aim of the current study was to evaluate the patterns of treatment and clinicopathology figures in Kurdish patients with TNBC, and to compare these to other studies.

\section{Materials and Methods}

\section{Patients}

Between of 2001 to 2014,950 breast cancer patients referred to Our Clinic, Kermanshah City, Iran. There were 74 female patients with TNBC that type of pathology in 70 patients was invasive ductal carcinoma and we entered them in our study. ER and PR positive were defined as positive immunohistochemical staining in more than $10 \%$ of tumor cells. HER-2 status was determined according to the guideline recommended by College of American Pathologists. Immunohistochemistry assay with anti-HER2 antibodies was used to identify HER negative $(0$ and $1+)$ or positive $(2+$ and $3+)$. HER2 gene amplification was determined by fluorescent in situ hybridization (FISH). Tumors with a positive FISH result were considered as HER2 positive. Tumors negative for ER, PR and HER2 were considered as triple-negative ( $\mathrm{Li}$ et al., 2013). Clinicopathology figures in the patients were calculated. Also, affection of tamoxifen and radiotherapy on OS of the patients was measured. The OS period was defined as the time diagnosis to the date of death or loss of the follow-up and patients alive with less than one year follow-up were deleted in our study. We calculated the OS for 3-year, 5-year and 10-year. Dose of tamoxifen for the patients was $20 \mathrm{mg}$ per day.

\section{Statistical analysis}

The Figures for the OS were plotted with GraphPad Prism 5 Software with using Kaplan-Meier and Log-rank testes for comparison of results. IBM SPSS Statistics 19 Software was used for the relationship of between of type of treatment and the OS (Chi-square Test), that $\mathrm{P}<0.05$ was statistically significant for results.

\section{Results}

The mean age in the first diagnosis for 70 patients with TNBC and invasive ductal carcinoma was 49.6 years $( \pm 12.6)$ that range of age was $27-82$ years and all of the patients were female. We divided to five age groups that more percentages of patients were in ranges of 40-49, 50$59,20-39,60-69$ and $\geq 70$, respectively. Of 70 patients, 23 patients had metastasis for organs that 8 patients $(37.8 \%)$ to bone, 5 patients $(21.8 \%)$ to lung, 4 patients $(17.4 \%)$ to liver, 2 patients $(8.6 \%)$ to brain and rest of patients to mentioned two or three organs (other). Thirty- two patients $(45.7 \%)$ were treated with tamoxifen and 39 patients $(55.7 \%)$ with radiotherapy. Heredity (at least one of the relatives of patients with any cancer) in triple negative breast cancer patients was 6 patients $(8.6 \%) .44$ patients $(62.9 \%)$ of patients had left breast tumor and 26 patients (37.1) had right breast tumor. Histological grade in the patients was included grade I: $5.7 \%$, grade II: $52.9 \%$ and grade III: $41.4 \%$. (Table 1 )

We measured enzymes aspartate transaminase (AST) and alanine transaminase (ALT) in the blood of patients that results showed 21 patients $(30 \%)$ had fatty liver. In TNBC patients, $14(20 \%)$ of them had tumors smaller than $2 \mathrm{~cm}, 48(68.6 \%)$ patients had tumors with size between 2 to $5 \mathrm{~cm}$, and $8(11.4 \%)$ patients had tumors larger than $5 \mathrm{~cm}$. Lymph node invasive in 37 patients $(52.8 \%$ ) was positive. (Table 1)

The Figure 1 shows 3-year, 5-year and 10-year OS for all of patients with TNBC and invasive ductal carcinoma. 3-year, 5-year and 10-year mean survival for the patients were 29, 39 and 57 months and survival rate was $82 \%$, $72 \%$ and $64 \%$, respectively.

The Figure 2 shows 3-year, 5-year and 10-year OS for

Table 1. Characteristics in Women with Triple Negative Breast Cancer and Invasive Ductal Carcinoma (N=70)

\begin{tabular}{|c|c|c|c|}
\hline Characteristics & $\mathrm{N}(\%)$ & Mean \pm SD & Range \\
\hline$\overline{\text { Age(year) }}$ & & $49.6 \pm 12.6$ & $27-82$ \\
\hline $20-39$ & $16(22.9)$ & & \\
\hline $40-49$ & $20(28.6)$ & & \\
\hline $50-59$ & $18(25.7)$ & & \\
\hline $60-69$ & $11(15.7)$ & & \\
\hline$\geq 70$ & $5(7.1)$ & & \\
\hline \multicolumn{4}{|l|}{ Metastasis } \\
\hline Yes & $23(32.9)$ & & \\
\hline No & $47(67.1)$ & & \\
\hline \multicolumn{4}{|c|}{ Type of metastasis $(\mathrm{n}=23)$} \\
\hline Bone & $8(34.8)$ & & \\
\hline Lung & $5(21.8)$ & & \\
\hline Liver & $4(17.4)$ & & \\
\hline Brain & $2(8.6)$ & & \\
\hline Other & $4(17.4)$ & & \\
\hline \multicolumn{4}{|l|}{ Tamoxifen } \\
\hline Yes & $32(45.7)$ & & \\
\hline No & $38(54.3)$ & & \\
\hline \multicolumn{4}{|l|}{ Radiotherapy } \\
\hline Yes & $39(55.7)$ & & \\
\hline No & $31(44.3)$ & & \\
\hline \multicolumn{4}{|c|}{ Family history of any cancer } \\
\hline Yes & $6(8.6)$ & & \\
\hline No & $64(91.4)$ & & \\
\hline \multicolumn{4}{|l|}{ Laterality } \\
\hline Right Breast & $26(37.1)$ & & \\
\hline Left Breast & $44(62.9)$ & & \\
\hline \multicolumn{4}{|c|}{ Histological grade } \\
\hline I & $4(5.7)$ & & \\
\hline II & $37(52.9)$ & & \\
\hline III & $29(41.4)$ & & \\
\hline \multicolumn{4}{|l|}{ Fatty Liver } \\
\hline Yes & $21(30.0)$ & & \\
\hline No & $49(70.0)$ & & \\
\hline \multicolumn{4}{|c|}{ Size of tumor in diameter $(\mathrm{cm})$} \\
\hline $0.1-2$ & $14(20.0)$ & & \\
\hline $2.1-5$ & $48(68.6)$ & & \\
\hline$>5$ & $8(11.4)$ & & \\
\hline \multicolumn{4}{|c|}{ Lymph node invasive } \\
\hline Yes & $37(52.8)$ & & \\
\hline No & $33(47.2)$ & & \\
\hline \multicolumn{4}{|l|}{ Chemotherapy } \\
\hline Yes & $70(100)$ & & \\
\hline No & $0(0.0)$ & & \\
\hline
\end{tabular}


Table 2. Patient and Tumor Characteristics for Triple Negative Breast Cancer

\begin{tabular}{|c|c|c|c|c|c|}
\hline References & Patients & $\begin{array}{l}\text { Mean Age } \\
\text { (year) }\end{array}$ & $\begin{array}{l}\text { Range of Age } \\
\text { (year) }\end{array}$ & $\begin{array}{l}\text { Histological Grade } \\
\text { (I-II-III) }\end{array}$ & $\begin{array}{l}\text { Lymph Node invasive } \\
\qquad(\%)\end{array}$ \\
\hline 7 & 30 & 44.9 & - & $(0-16.7-83.3)$ & 51.9 \\
\hline 8 & 111 & 63 & $28-81$ & $(22-40-38)$ & 74 \\
\hline 9 & 256 & 51 & $20-78$ & $(0.8-13.7-85.5)$ & - \\
\hline 2 & 67 & 48 & - & $(1.5-28.4-70.1)$ & 40.3 \\
\hline 10 & 40 & 60.3 & - & $(0-28.2-71.8)$ & - \\
\hline 11 & 132 & 52.3 & - & $(6.2-53.5-40.3)$ & 50.8 \\
\hline 12 & 33 & 55.9 & - & $(3.3-16.7-80)$ & - \\
\hline 13 & 43 & 50 & - & - & 40 \\
\hline 14 & 269 & 55 & $23-88.5$ & $(2.7-14.8-82.5)$ & - \\
\hline 16 & 180 & 53 & $22-92$ & $(19.9-51.6-28.5)$ & - \\
\hline 19 & 194 & 39.4 & $19-71$ & $(3.6-18.6-77.8)$ & - \\
\hline 20 & 107 & 46.7 & - & $(20.8,17.8,79.4)$ & 57.9 \\
\hline
\end{tabular}

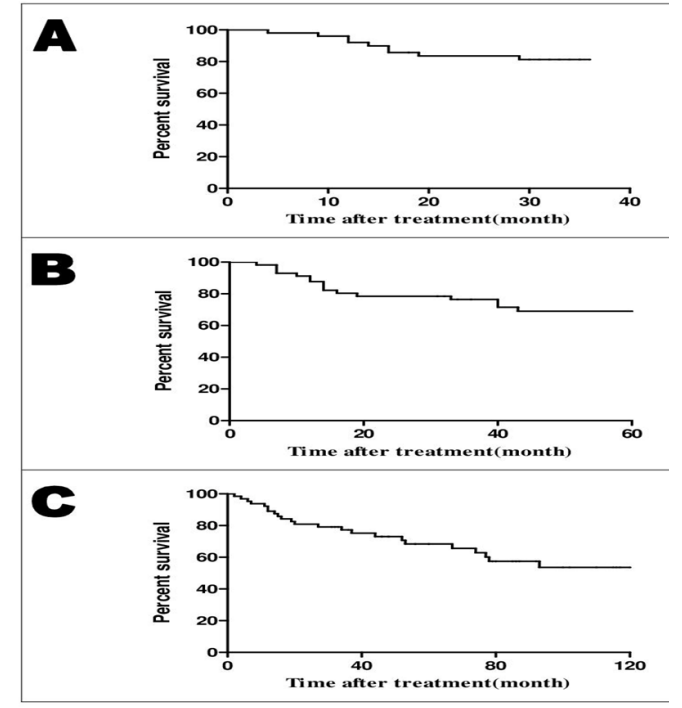

Figure 1. Overall Survival for Patients with Triple Negative Breast Cancer and Invasive Ductal Carcinoma. (A) 3- year overall survival; (B) 5-year overall survival; (C) 10-year overall survival

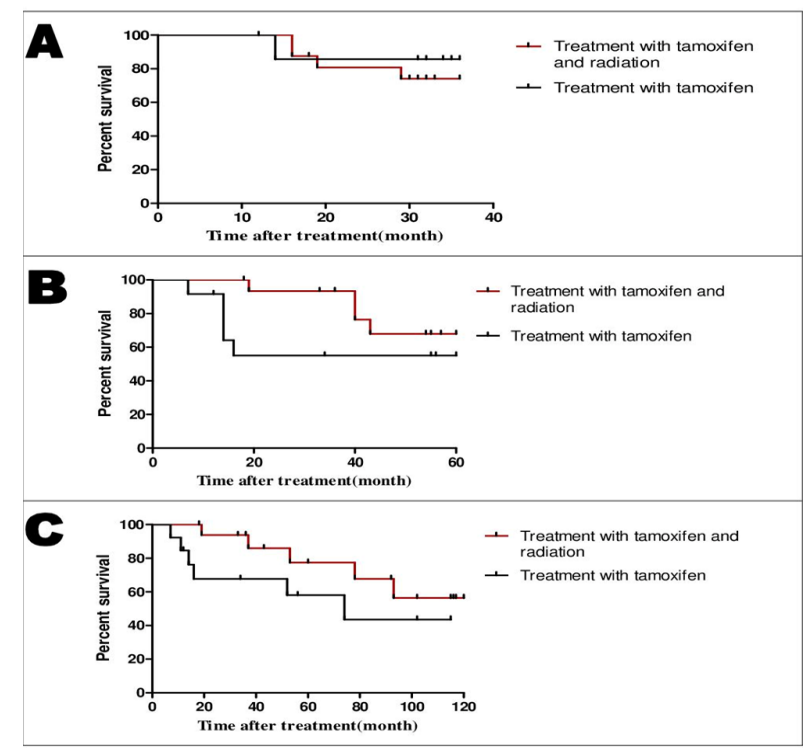

Figure 2. Overall Survival for Patients with Triple Negative Breast Cancer and Invasive Ductal Carcinoma that Treated with Tamoxifen Plus Radiation Compared to Tamoxifen. (A) 3- year overall survival; (B) 5-year overall survival; (C) 10-year overall survival

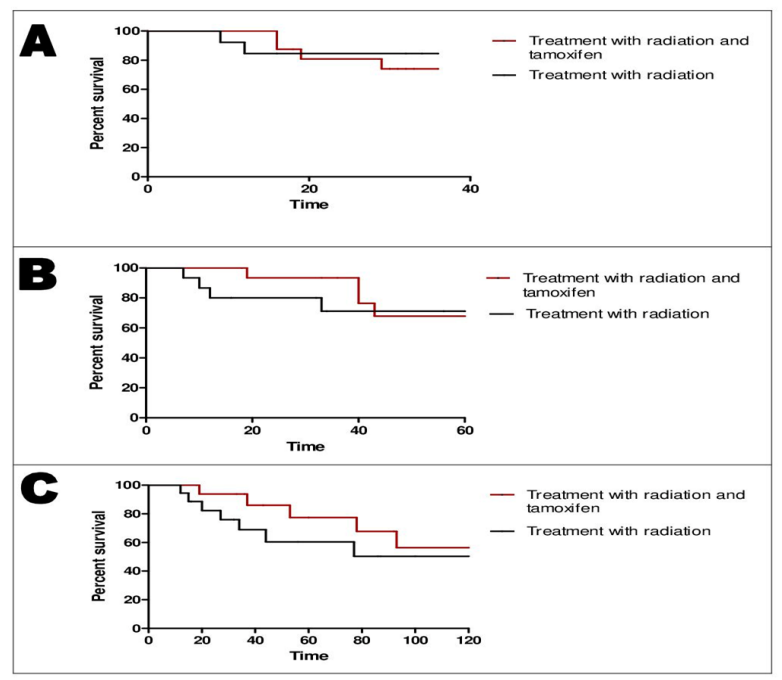

Figure 3. Overall Survival for Patients with Triple Negative Breast Cancer and Invasive Ductal Carcinoma that Treated with Tamoxifen Plus Radiation Compared to Radiation. (A) 3- year overall survival; (B) 5-year overall survival; (C) 10-year overall survival

TNBC patients that treated with tamoxifen plus radiation compared to tamoxifen alone. 3-year, 5-year and 10-year mean survival for treated patients with tamoxifen plus radiation was $29.4,44.5$ and 68.5 months and survival rate were $75 \%, 75 \%$ and $70.6 \%$, respectively. 3 -year, 5-year and 10-year mean survival for treatment with tamoxifen alone was $28.3,32.8$ and 51 and survival rate was $87.5 \%, 58.3 \%$ and $53.8 \%$, whereas mean survival for treatment with radiation alone (Figure 3) was 27.5, 36.1 and 51.5 months and survival rate was $84.6 \%, 73.3$ and $61.1 \%$, respectively. There was no significant relationship between OS for treated patients with tamoxifen plus radiation compared to tamoxifen or radiation alone $(\mathrm{P}>0.05)$.

\section{Discussion}

Breast cancer screening and higher quality mammography have resulted in an increase in the diagnosis of ductal carcinoma in situ worldwide (Payandeh et al., 2014). TNBC is only partially responsive to chemotherapy and presents a lack of clinically established targeted therapies. TNBC accounts for $10-17 \%$ of all breast 
carcinomas (Dogra et al., 2014). The Table 2 shows a number of studies that surveyed characteristics of patients with TNBC. Based on these studies, due to the large number of breast cancer in treatment centers and clinics, number of patients with TNBC is low, for example in this study, out of 950 patients with breast cancer 74 patients were TNBC (12.8\%) and also based on information from previous studies, invasive ductal carcinoma is the most subgroup of breast cancer. Our study showed that out of 74 patients with TNBC, 70 patients had invasive ductal carcinoma(94.6\%). the mean age for TNBC is between 40 to 65 years with a focus on 45-55 years such as mean age in our study that was 49.6 years. Also, In our study, range of age was 27-82 years that studies (Dent et al., 2009; Ovcaricek et al., 2011; Bayoumi et al., 2014; Kim et al., 2015) resulted the range is between 19-92 years. Histological grade for the patients consist of three grades that grade I in more studies on TNBC patients (Table 2) and also our study was less than $7 \%$. In more studies, results showed that grade III in TNBC patients is more than grade II, but this study is contrasting with these results and similar to results of two other studies (Dent et al., 2009; Somali et al., 2013; Bayoumi et al., 2014). At least, lymph node invasive in $50 \%$ of TNBC patients is positive that our study confirms it.

A study (Li et al., 2013) on 2649 TNBC patients showed that tumor size in them is: $20.91 \% \leq 2 \mathrm{~cm}, 25.94 \%$ between $2-5 \mathrm{~cm}$ and $53.15 \% \geq 5 \mathrm{~cm}$ and also in other study (Dent et al., 2009) tumor size was $36.5 \% \leq 2 \mathrm{~cm}, 55.6 \%$ between $2-5 \mathrm{~cm}$ and $7.9 \%>5 \mathrm{~cm}$. In a study (Dogra et al., 2014 ) on 67 patients it was $6 \%<2 \mathrm{~cm}, 70.1 \%$ between of $2-5 \mathrm{~cm}$ and $23.9 \%>5 \mathrm{~cm}$, similar to our study on $70 \mathrm{TNBC}$ patients that was $20 \%, 68.6 \%$ and $11.4 \%$, respectively. These results show that tumor size in more studies for TNBC is equal to $x(2 \leq x \geq 5)$. Based on two studies ( $\mathrm{Li}$ et al., 2013; Kim et al., 2015), family history of breast cancer in TNBC patients was $34.84 \%$ and $10.2 \%$, whereas in our study, family history of any cancer was in 6 patients $(8.6 \%)$ and 1 patients $(1.4 \%)$ had family history of breast cancer. Therefore, we conclude that in our area for study (West Iran), family history have less impact on TNBC patients from other area.

In a study (Dey et al., 2013) has been written that TNBC is a highly metastatic disease, but study (Li et al., 2013) reported that metastasis in TNBC patients is $20.12 \%$ that among those TNBC patients with a metastatic cancer, the cancer was metastasized to the lungs in $41.8 \%$, to the liver in $42.8 \%$, to the brain in $9 \%$ patients, and to the bones in $6.4 \%$ patients. Overall, metastatic TNBC had a higher rate of metastasis to the lungs and liver than to the brain and bones and in our study, metastasis in TNBC patients was $32.9 \%$ that metastatic TNBC had a higher rate of metastasis to bone, lung, liver and brain, respectively. Therefore, TNBC isn't a highly metastatic disease.

Side of involvement in a study (Salami et al., 2011) was $40 \%$ for right breast, and other study (Dogra et al., 2014) reported $52.2 \%$ patients had right involvement. This study showed that right involvement is $37.1 \%$ patients. Overall, left breast involvement is more than left breast in TNBC patients.

The 5-year OS was $74.5 \%$ for TNBC patients, also recurrences and death rates were higher only in the first 3 years following the diagnosis (Ovcaricek et al., 2011). The 5-year OS rates for TNBC patients was 79.92\% and The 7-year overall survival rates was $71.64 \%$ ( Li et al., 2013). In our study, 3-year, 5-year and 10-year OS were $82 \%, 72 \%$ and $64 \%$, respectively. Also, in patients with TNBC in a London population, the 5-year survival was $62 \%$ (Pal et al., 2014) and in other research was $78 \%$ (Bhatti et al., 2014) and in center of Iran was 90\%.(20) These results showed that OS in West Iran (our study) is less than other studies except to London that was very less. Patients with TNBC derive no benefit from molecularly targeted treatments such as endocrine (tamoxifen or letrozole) therapy or trastuzumab, because they don't have the appropriate targets for these drugs (Liedtke et al., 2008). Nevertheless, a small percentage of TNBC patients can respond to treatment with tamoxifen (Dent et al., 2009). Also, TNBC is associated with poor OS (Pal et al., 2014). In our study, 3-year, 5-year and 10year OS for treated patients with radiation or tamoxifen alone were $84.6 \%, 73.3$ and $61.1 \%$ or $87.5 \%, 58.3 \%$ and $53.8 \%$, respectively, but for treated patients with combination of tamoxifen plus radiation were $75 \%, 75 \%$ and $70.6 \%$. A study (Steward et al., 2014) demonstrated that TNBC patients who underwent radiation therapy had significantly improved overall survival compared with those who did not receive adjuvant radiation therapy. The overall four-year survival for patients who received adjuvant radiation therapy was $77.34 \%$ versus $59.8 \%$ in patients who did not receive adjuvant radiation therapy. Based on our study, treatment with radiation is better than tamoxifen in TNBC patients but our study for the first time showed that treatment for TNBC patients with combination of tamoxifen plus radiation increase the OS and also reduced mortality rate after 3 years from the first diagnosis and even is better than treatment with radiation alone after 5 years from the first diagnosis for breast cancer particularly in 10-year period. The correlation between the treatments in 3-year and 5-year and 10-year periods was not statistically significant $(\mathrm{P}>0.05)$.

In summary, factors (Table 1) such as number of patients with metastasis and high grade and also chemotherapy (number of treated patients and type) and radiotherapy (number of treated patients and type) can affect on survival in the patients.

In conclusion, first, it can result that tumor size in more studies for TNBC is equal to $\mathrm{x}(2 \leq \mathrm{x} \geq 5)$. Second, the OS in West Iran (our study) is less than a lot of studies. At last, treatment for TNBC patients with combination of tamoxifen plus radiation increases the OS and reduces mortality rate.

\section{References}

Aghili M, Lashkari M, Farrokhpey AH, et al (2013). Triplenegative breast cancer survival in Iranian patients. Acta Med Iran, 51, 560-6.

Bayoumi Y, AbdelSamie A, Abdelsaid A, et al (2014). Locoregional recurrence of triple-negative breast cancer: effect of type of surgery and adjuvant postoperative radiotherapy. Breast Cancer (Dove Med Press), 6, 151-8.

Bhatti AB, Khan AI, Siddiqui N, et al (2014). Outcomes of triple- 
negative versus non-triple-negative breast cancers managed with breast-conserving therapy. Asian Pac J Cancer Prev, 15, 2577-81.

Carey LA (2011). Directed therapy of subtypes of triple-negative breast cancer. Oncologist, 16, 71-8.

Dent R, Hanna WM, Trudeau M, et al (2009). Pattern of metastatic spread in triple-negative breast cancer. Breast Cancer Res Treat, 115, 423-8.

Dey N, Barwick BG, Moreno CS, et al (2013). Wnt signaling in triple negative breast cancer is associated with metastasis. BMC Cancer, 13, 537.

Dogra A, Doval DC, Sardana M, et al (2014). Clinicopathological characteristics of triple negative breast cancer at a tertiary care hospital in India. Asian Pac J Cancer Prev, 15, 1057783.

Foulkes WD, Smith IE, Reis-Filho JS (2010). Triple-negative breast cancer. $N$ Engl J Med, 363, 1938-48.

Hudis CA, Gianni L (2011). Triple-negative breast cancer: an unmet medical need. Oncologist, 16,1-11.

Kim WH, Han W, Chang JM, et al (2015). Location of TripleNegative Breast Cancers: Comparison with Estrogen Receptor-Positive Breast Cancers on MR Imaging. PLoS One, 10, 0116344.

Liedtke C, Mazouni C, Hess KR, et al (2008). Response to neoadjuvant therapy and long-term survival in patients with triple-negative breast cancer. J Clin Oncol, 26, 1275-81.

Li CY,Zhang S, Zhang XB, et al (2013). Clinicopathological and prognostic characteristics of triple- negative breast cancer (TNBC) in Chinese patients: a retrospective study. Asian Pac J Cancer Prev, 14, 3779-84.

Ovcaricek T, Frkovic SG, Matos E, et al (2011). Triple negative breast cancer - prognostic factors and survival. Radiol Oncol, 45, 46-52.

Pal S, Lüchtenborg M, Davies EA, et al (2014). The treatment and survival of patients with triple negative breast cancer in a London population. Springerplus, 3, 553.

Payandeh M, Sadeghi M, Fekri A, et al (2014). P53 mutation compared with Ki67 marker in metastasis of breast cancer in western Iran. J Solid Tumors, 4, 4-9.

Payandeh M, Sadeghi M, Sadeghi E, et al (2014). Comparison of IHC, FISH, ER and PR in Breast Cancer in Western Iran. Am J Cancer Prev, 2, 37-41.

Piana A, Sotgiu G, Muroni M, et al (2014). HPV infection and triple-negative breast cancers: an Italian case-control study. Virol J, 11, 190.

Salami S, Ramezani F, Aghazadeh T, et al (2011). Impact of triple negative phenotype on prognosis and early onset of breast cancer in Iranian females. Asian Pac J Cancer Prev, 12, 719-24.

Sharma M, Sharma JD, Sarma A, et al (2014). Triple negative breast cancer in people of North East India: critical insights gained at a regional cancer centre. Asian Pac J Cancer Prev, 15, 4507-11.

Sioshansi S, Ehdaivand S, Cramer C, et al (2012). Triple negative breast cancer is associated with an increased risk of residual invasive carcinoma after lumpectomy. Cancer, 118, 3893-8.

Somali I, Ustaoglu BY, Tarhan MO, et al (2013). Clinicopathologic and demographic evaluation of triple- negative breast cancer patients among a turkish patient population: a single center experience. Asian Pac J Cancer Prev, 14, 6013-7.

Stevens KN, Vachon CM, Couch FJ (2013). Genetic susceptibility to triple-negative breast cancer. Cancer Res, 73, 2025-30.

Steward LT, Gao F, Taylor MA, et al (2014). Impact of radiation therapy on survival in patients with triple-negative breast cancer. Oncol Lett, 7, 548-552.

Tan GH, Taib NA, Choo WY, et al (2009). Clinical characteristics of triple-negative breast cancer: experience in an Asian developing country. Asian Pac J Cancer Prev, 10, 395-8.

Wojcinski S, Soliman AA, Schmidt J, et al (2012). Sonographic features of triple-negative and non-triple-negative breast cancer. J Ultrasound Med, 31, 1531-41. 